\title{
Una Propuesta para Fortalecer el Énfasis Profesional del Currículo de Ingeniería Química
}

\author{
Fabio Castrillón, Erika Arenas, Dora Carmona, Beatriz Garcés \\ Facultad de Ingeniería Química, Universidad Pontificia Bolivariana, Circular 1 No. 70 - 01, Medellín, \\ Colombia (e-mail: fabio.castrillon@upb.edu.co; erika.arenas@upb.edu.co; dora.carmona@upb.edu.co; \\ beatriz.garces@upb.edu.co)
}

Recibido Abr. 29, 2015; Aceptado Jun. 3, 2015; Versión final Ago. 1, 2015, Publicado Feb. 2016

\begin{abstract}
Resumen
Se describe una propuesta para fortalecer el énfasis profesional del currículo de ingeniería química en la Universidad Pontificia Bolivariana (UPB) en Colombia. Tanto las áreas del saber como los campos de acción asociados a la ingeniería química han variado y se han multiplicado en las últimas décadas, impulsando al mundo académico a renovar el proceso de formación de los futuros profesionales. Es por ello que la Facultad de Ingeniería Química de la UPB ha diseñado una estrategia que, mientras especializa al estudiante en un campo específico del conocimiento y el desempeño de la ingeniería química, vincula la formación profesional con la de postgrado. Se trata de un conjunto de 15 créditos, denominado Ruta de Formación Optativa, donde 6 créditos corresponden a cursos de carrera y 9 créditos a cursos de postgrado de la Escuela de Ingenierías. Se presentan las nueve rutas diseñadas, que configuran nueve perfiles profesionales y ocupacionales específicos, entre los cuales el estudiante puede elegir, fortaleciendo su autonomía, a la vez que se promueve y se facilita su educación de alto nivel.
\end{abstract}

\section{A Proposal to Strengthen the Professional Emphasis in a Chemical Engineering Undergraduate Curriculum}

\begin{abstract}
A proposal to strengthen the professional emphasis in the chemical engineering undergraduate curriculum at the Universidad Pontificia Bolivariana (UPB) in Colombia is presented. In recent decades, the areas of knowledge and the professional fields associated to chemical engineering have changed and increased greatly, encouraging the renovation of the undergraduate formation process. The Chemical Engineering Program of UPB has designed a strategy to bring specialized training in specific fields of knowledge and professional performance to students, integrating undergraduate and graduate education. This strategy, called Optional Training Routes, consists of a combination of 15 elective credits, of which 6 correspond to undergraduate courses and 9 to graduate courses offered by the School of Engineering. This paper shows the nine optional training routes designed, which configure nine professional profiles and promote further education at graduate level. Undergraduate students can choose one of these routes according to their individual skills and interests, strengthening their own autonomy.
\end{abstract}

Keywords: chemical engineering; curricular design; undergraduate-graduate integration; undergraduate education 


\section{INTRODUCCIÓN}

En la última década se hizo evidente que el campo de acción de la ingeniería química ha experimentado una vasta ampliación, brindando una notable gama de nuevas oportunidades de desempeño a los graduados en la disciplina (Armstrong, 2006; Byrne, 2006; Charpentier, 2005; Molzahn, 2004). La ingeniería química ha pasado de estar ligada de manera muy estrecha a una industria en particular, la petroquímica, a ser cada vez más relevante en otras áreas industriales, como la electrónica, la alimentaria, la biotecnológica, la farmacéutica, la nanotecnológica, entre otras. Esto se ha debido, en gran parte, a la ubicación privilegiada de la ingeniería química, justo en la interfase entre las ciencias moleculares y la ingeniería. En cuanto al campo investigativo, la disciplina ha profundizado en la perspectiva molecular, ha continuado avanzando en el área de los materiales y ha incorporado muchas inquietudes del área biológica; tanto que Armstrong, ya en 2006, proponía que el ingeniero químico debía sentirse tan cómodo manipulando moléculas biológicas, como largas cadenas poliméricas sintéticas, y Gillett, desde 2001, enfatizaba las contribuciones de la bioquímica al futuro de la ingeniería química.

El cambio también ha tocado el tipo de productos y las características de los procesos que la ingeniería química está llamada a desarrollar y sostener. Cada vez más, haciéndose un considerable lugar al lado de los productos químicos básicos (commodities), prospera la demanda por productos químicos altamente especializados (specialty chemicals), lo que impone el desarrollo de procesos de producción de alta selectividad (Byrne, 2006; Charpentier, 2005; Gillett, 2001). Por su parte, la creciente preocupación pública por cuestiones medioambientales y de seguridad, ha exigido innovación en el diseño de procesos químicos sostenibles, de manera que tengan en cuenta la seguridad, la salud y el medio ambiente. Para esto, se ha practicado la integración de factores medioambientales, sociales y económicos en la evaluación de proyectos y diseños (Allen y Shonnard, 2012), y más en detalle, se ha puesto énfasis en las tecnologías de cero emisiones, la eficiencia energética (bajo consumo, reducción de pérdidas), la reducción del uso de materias primas no renovables y el aumento del reciclaje de productos y subproductos (Charpentier, 2005). Tocando el aspecto energético, Armstrong (2006) e IChemE (2007) señalan como tarea vital de la ingeniería química contribuir a la ampliación de la generación de energía a bajo costo, así como a su almacenamiento y transporte.

Autores como Charpentier (2005) y Armstrong (2006) indican que estos desafíos han fortalecido la necesidad de que el diseño de procesos y productos se realice de manera multidisciplinar, y a multiescala, tanto en tamaño como en tiempo; esto es, diseños que abarquen desde el nivel molecular hasta el del sitio de producción entero, y que se proyecten en variados rangos de tiempo. Sin duda, este tipo de prácticas exige grandes capacidades para la modelación y la simulación computacional. Efectivamente, el uso del computador ha permitido profundizar en la modelación de propiedades físicas desde la intervención de la estructura a escala nano y micro; esto ha hecho posible, por ejemplo, diseñar productos sólidos de formas y texturas altamente específicas, así como emulsiones especializadas; una de las áreas más favorecidas por estos desarrollos ha sido la de la catálisis.

El ámbito académico ha reconocido los anteriores retos y ha considerado la forma de responder a ellos. En los últimos años, se ha realizado una intensa revisión del proceso de formación universitaria en ingeniería a nivel mundial. Por ejemplo, en el marco de la educación del futuro ingeniero, han tomado protagonismo contenidos y actividades curriculares que fomenten su desarrollo de ciertas cualidades generales como el conocimiento y manejo de herramientas contemporáneas (tanto técnicas como informáticas), el adecuado desenvolvimiento en un trabajo en equipo y en los procesos de comunicación, y la capacidad de cultivar de manera permanente su mejoramiento profesional (Castrillón y Garcés, 2012).

Para la ingeniería química, después de la amplia revisión de literatura y otras fuentes que realiza Byrne (2006), el autor presenta un modelo de lo que sería, actualmente, la ingeniería química universal, donde el núcleo de la disciplina (operaciones unitarias, fenómenos de transporte, etc.) está acompañado por lo que llama opciones secundarias: química, biología (biotecnología, bioprocesos), industrias específicas (farmacéutica, plásticos, combustibles, alimentos, entre otras posibilidades), administración y negocios, medio ambiente, humanidades, aspectos legales, computación (incluido el control de procesos), habilidades profesionales (trabajo en equipo, comunicación, etc.). Estas opciones secundarias pueden variar mucho en cuanto a su número y su preponderancia en el currículo ofrecido por una universidad en particular, lo que depende de las decisiones de cada institución, ligadas a condiciones específicas de los países, las regiones y/o de la industria local. Por ejemplo, el autor señala que en los países en desarrollo tiende a predominar la necesidad de ingenieros químicos fuertes en el núcleo central de la disciplina, orientados a los procesos de producción masiva. Byrne muestra algunas de las formas que se han ideado diferentes universidades del mundo para ofrecer las opciones secundarias, las que incluyen la combinación de cursos de diferentes carreras, la opción de doble titulación y la posibilidad de tomar cursos de postgrado durante la realización de la carrera. 
En este panorama, teniendo en cuenta la multiplicación de las áreas tecnológicas que se tocan con la ingeniería química en la actualidad, Armstrong (2006) y Byrne (2006) han señalado que se abre un importante cuestionamiento acerca de la decisión más acertada para la formación profesional: ¿se deben enseñar muchas áreas particulares de la tecnología; o seleccionar algunas para crear especializaciones o énfasis específicos; o mantener un núcleo común de ingeniería química fuerte y dejar la especialización para el postgrado?

\section{LA FORMACIÓN EN INGENIERÍA QUÍMICA HOY}

Académicos como Armstrong (2006) han presentado propuestas curriculares donde el esfuerzo en la carrera de ingeniería química se dirige a fortalecer el núcleo común de la disciplina, en vez de proveer rutas de especialización; sin embargo, el autor no abandona la idea de ofertar algunos cursos electivos. En la otra orilla, Byrne (2006) reseña que la revista Chemical Engineering Progress publicó en el año 2002 un número especial titulado "An evolution in chemical engineering - The journey ahead", que incluía las propuestas de un grupo de académicos de Alemania, Gran Bretaña y Estados Unidos sobre los cambios concretos que podrían hacerse en el currículo tradicional de ingeniería química para dar cabida a diversos cursos sobre ciencia de los materiales (incluyendo biopolímeros), bioquímica, biología celular y emprendimiento de empresas. La alternativa incluye cambios tan polémicos como reducir la formación en termodinámica, control de procesos y optimización; eliminar el estudio de la mecánica cuántica y no considerar al diseño de procesos como el elemento integrador (capstone) del currículo. Los mismos autores de la propuesta declararon que ésta no es -y no debería ser- del agrado de la mayor parte del mundo académico de la ingeniería química. Por último, dichos académicos ofrecen un plan más asequible: ofrecer tres rutas de especialización en ingeniería química, una en producción de químicos básicos, otra en producción de químicos especiales, y otra con énfasis en la sostenibilidad.

Retomando el análisis de la opción de abarcar muchas especialidades, o tecnologías específicas, en un mismo currículo para ingeniería química, Armstrong establece que esta opción, además de difícil de realizar porque se dispone de tiempo limitado, tendería a fragmentar la disciplina. Pero es tan atractiva la iniciativa de abarcar la creciente diversidad, que Molzahn (2004) declara que hay que tener coraje para no tratar de incluirlo todo en un mismo plan de estudios. Crawley (2007), en un texto sobre el marco para la educación en ingeniería que se conoce como CDIO (Concebir - Diseñar - Implementar - Operar), señala otros riesgos de introducir en el currículo muchas especializaciones originadas por los progresivos avances en las ciencias que nutren la carrera: pueden llevar a la ingeniería lejos del conocimiento práctico que necesita y la caracteriza. Esto se debe a que muchas de estas nacientes disciplinas se pretenden enseñar en cursos individuales, donde el énfasis no está centrado en sus aplicaciones. De este modo, no contribuyen realmente al conjunto de competencias en ingeniería, pues se apartan de su dominio primordial: solucionar problemas prácticos.

La opción intermedia, consistente en diseñar currículos profesionales para ingeniería química que le den espacio suficiente al núcleo de la disciplina, pero que complementen la educación con algunos énfasis en tecnologías específicas, ha sido bastante acogida a nivel mundial. Académicos como Byrne (2006) manifiestan que algunas compañías podrían no estar muy interesadas en profesionales que solo estén formados en la producción de químicos a gran escala, y preferirían un graduado con una educación especializada en un área de interés para su sector industrial. El apoyo a esta opción curricular no solo provendría del mundo industrial, en 2004 O’Neill (citado por Byrne, 2006) realizó un estudio que consignaba la opinión de jefes de los departamentos de ingeniera química de diversas universidades europeas, quienes coincidieron en la importancia de una flexibilidad curricular que diera a los estudiantes la oportunidad de abordar tanto los cursos tradicionales como tópicos relacionados con las nuevas industrias.

Por su parte, Gillett (2001) expresa que los cursos electivos a ofrecerse en un plan de estudios pueden provenir de los énfasis investigativos de la institución particular (sus grupos de investigación, líneas, proyectos). Este autor enfatiza que la ingeniería química, en un panorama tan cambiante como el actual, exige una formación que no finaliza con la graduación; se estima que la vida media del conocimiento en ingeniería química es apenas de 4 a 5 años; de este modo un ingeniero químico debería dedicar por lo menos el $10 \%$ de su tiempo a su actualización, para evitar la obsolescencia. El autor indica que los estudios de postgrado son la puerta de entrada para desarrollar la capacidad de aprendizaje para toda la vida. Este panorama fundamenta la decisión de ofrecer la posibilidad de que los estudiantes de carrera tomen cursos de postgrado, ya sea bajo la forma de cursos electivos o como parte de una ruta de especialización.

En relación a las tecnologías, o tipos de industria, que sería indicado incorporar en todo currículo de la carrera, Byrne (2006) advierte que el mundo académico de la ingeniería química se encuentra lejos de un consenso. Sin embargo, se han detectado intereses mayoritarios, como la biotecnología y el área del medio ambiente y la sostenibilidad. 
En cuanto al tema de la sostenibilidad, Allen y Shonnard (2012) presentan un cuerpo de conocimientos básico para incorporar su comprensión y manejo en la educación en ingeniería química, donde se entrega a los estudiantes herramientas para que puedan cumplir los objetivos básicos de aumentar la eficiencia energética y reducir el uso de materiales y las emisiones de los procesos. Los autores también indican que, actualmente, estos conocimientos se ofrecen en la inmensa mayoría de los programas de ingeniería de los Estados Unidos, usualmente bajo la forma de cursos electivos, pero subrayan que también pueden incorporarse en los cursos obligatorios del currículo, y presentan propuestas para hacerlo.

Por su parte, Cortese (2003), recalcando la importancia de la sostenibilidad, sugiere una educación donde los contenidos y contextos de aprendizaje se basan en sistemas interdisciplinarios y dinámicos, cuyo tema central es la interdependencia ser humano/medio ambiente, los valores y la ética. Como ejemplo de la incorporación del diseño ambientalmente sostenible en un currículo, el autor presenta el caso la universidad norteamericana Carnegie Mellon, que ofrece a todos sus estudiantes un curso de introducción al tema medioambiental, así como cursos electivos que permiten una comprensión más profunda de temas científicos, ingenieriles, económicos, sociales y políticos relacionados con el ambiente.

Respecto a la biotecnología, Assaf-Anid y Hollein (2002) muestran que, mientras algunos programas profesionales de ingeniería química se limitan a ofrecerlo como un tema de libre elección, otros, como el del Manhattan College de Estados Unidos, incluyen en su plan de estudios un curso obligatorio en el área de biotecnología, al tiempo que brindan la posibilidad de que los estudiantes tomen cursos electivos adicionales en el área, tales como ingeniería de bioreacciones, ingeniería de bioseparación, microbiología industrial, procesos de tratamiento biológico, entre otros. Estos están abiertos tanto para estudiantes de los últimos años de la carrera, como para estudiantes de postgrado.

En concordancia con todo lo referido, un estudio comparado patrocinado por la Organización de los Estados Americanos OEA, entre las carreras de ingeniería química de nueve universidades de la región, permite apreciar que los temas más frecuentes en la oferta de especialización que contemplan son los relativos al medio ambiente y los materiales, seguidos por los relativos a la energía, la biotecnología y la bioingeniería (Carmona, 2014). Por su parte, en una investigación sobre los principales elementos del currículo de ingeniería química en varias universidades públicas del centro y sur de México, León y Lugo (2015) destacan temas emergentes en el perfil profesional, recogidos en tres áreas de énfasis: Procesos, Ciencias de los materiales y Ciencias ambientales. Reportan que en tales áreas se profundiza en tópicos específicos como diseño de producto, optimización de cadenas de suministro, ciclo de vida, energía y desarrollo sostenible, reciclaje y subproductos, biotecnología, polímeros y nuevos materiales.

Atendiendo el panorama anteriormente presentado, la Facultad de Ingeniería Química de la Universidad Pontificia Bolivariana (UPB) llevó a cabo recientemente una transformación curricular de la carrera, alineada con la opción de conservar una formación en el núcleo común de la disciplina en combinación con el ofrecimiento de rutas de especialización; la propuesta curricular cuenta con el desarrollo de competencias en diseño de procesos como el elemento integrador. La estrategia planeada brinda al estudiante la posibilidad de elegir un área de especialización profesional, y de vincularse con un postgrado en la misma área, desde antes de terminar su carrera (llamada carrera o programa de pregrado en varios países latinoamericanos, como Colombia, y carrera de grado en algunos otros países, como Argentina). En el presente artículo se expone la estrategia diseñada.

Para contextualizar la transformación curricular llevada a cabo, se informa que la propuesta que se publica en este artículo fue elaborada por el Comité de currículo de la Facultad de Ingeniería Química, integrado por los autores de este trabajo, en el marco de los lineamientos brindados por la UPB (2011a; 2011b; 2011c) para la transformación curricular de toda su oferta académica. La nueva propuesta curricular para ingeniería química, completa, puede consultarse en el artículo de Castrillón y Garcés (2012). Cabe resaltar que los lineamientos institucionales de la UPB establecen que la formación se basa en el desarrollo de capacidades humanas y competencias, y que el currículo se ciñe a cinco principios: contextualización, flexibilidad, interdisciplinariedad, integración e interculturalidad. A nivel curricular, el ofrecimiento de diversas rutas de especialización para los estudiantes de ingeniería química es una estrategia que favorece el desarrollo de competencias profesionales pertinentes, a la vez que fortalece la flexibilidad, la interdisciplinariedad y la integración del currículo.

\section{FLEXIBILIDAD E INTEGRACIÓN PREGRADO-POSTGRADO EN LA UPB}

La iniciativa de proveer al estudiante posibilidades de integrar pregrado y postgrado ya se encuentra recogida en las propuestas del Modelo Pedagógico de la UPB (2009b), donde se señala a la flexibilidad como una de las características de todos los currículos que se ofrecen. Uno de los aspectos de dicha flexibilidad es la apertura de los límites y las relaciones entre los ciclos y las áreas académicas. Es 
importante aclarar que los planes de estudio de los programas de pregrado en la UPB cuentan con cuatro ciclos: Básico de Formación Humanista, Básico Disciplinar, Profesional y de Integración. Mientras el Ciclo Profesional (CP) forma en los aspectos propios de la profesión, el reconocimiento del contexto de la misma y los posibles campos de desempeño; el Ciclo de Integración (Cl) está dedicado a abrir el proceso de la formación avanzada, e implica tomar cursos pertenecientes a algún postgrado. La estrategia presentada en este trabajo propende no solo por fortalecer las relaciones entre pregrado y postgrado, sino por afianzar la relación entre los ciclos $\mathrm{CP}$ y $\mathrm{Cl}$ del pregrado, ampliando el espacio donde el estudiante pone en juego su capacidad de decisión para seleccionar y combinar los componentes y las secuencias de su formación. Esta estrategia lleva por nombre Rutas de Formación Optativas.

\section{Componentes de las rutas de formación optativas}

Las rutas de formación optativas consisten en un conjunto de 15 créditos $(9 \%$ del total de créditos de la carrera), donde 6 de ellos corresponden al Ciclo Profesional, y los restantes 9 corresponden al Ciclo de Integración. Estos conjuntos marcan una ruta que especializa la formación del estudiante en función de sus intereses y capacidades, y sus componentes se convierten en obligatorios desde el momento en que el estudiante escoge una determinada ruta. Conviene precisar que el crédito es la medida del trabajo académico del estudiante de educación superior en Colombia, de manera que cada crédito corresponde a 48 horas de dedicación en total, lo que incluye tanto las horas de clase como las de trabajo autónomo, realizado por fuera de clase (República de Colombia, 2010).

Para detallar los componentes de las rutas, es importante comenzar por señalar que los créditos del CP que las conforman reciben el nombre de créditos optativos, y tienen por intención que el estudiante profundice en conocimientos y habilidades pertinentes para el ejercicio de la ingeniería química en un nicho específico del medio laboral. Algunos de los cursos que pueden cubrir estos créditos serán ofrecidos por la misma Facultad de Ingeniería Química. Aumentando la flexibilidad de la iniciativa, estos créditos también pueden ser cubiertos con cursos ofrecidos por otras facultades de la Escuela de Ingenierías que versen sobre tópicos y capacidades igualmente valiosas para la formación de un ingeniero químico.

Por otra parte, todos los créditos que conforman el $\mathrm{Cl}$ son optativos, y como se ha dicho, permiten que el estudiante de pregrado realice una introducción a los estudios de postgrado tomando cursos de formación avanzada, de manera que se facilite su paso a este nivel educativo, si él mismo lo decide luego de su graduación. Vale aclarar que los créditos de postgrado les serán reconocidos a los estudiantes como aprobados para su plan de estudios de pregrado en todos los casos, desde que cumplan los requisitos regulares de estos cursos (por ejemplo, en la UPB los cursos de pregrado se aprueban si se obtiene una nota definitiva igual o superior a 3.0 sobre 5.0 ); pero estos mismos créditos les serán reconocidos a los estudiantes como aprobados para un pensum de postgrado si, además, al cursarlos también cumplen con los requisitos establecidos para los cursos de este nivel (por ejemplo, para maestría los cursos se aprueban con una calificación definitiva igual o superior a 3.5 sobre 5.0 ).

\section{METODOLOGÍA}

Para definir los conjuntos específicos de cursos que conformarían la oferta de rutas de formación optativas para el pregrado, el Comité de currículo de la Facultad de Ingeniería Química de la UPB inicialmente estableció los campos profesionales en los cuales sería pertinente la especialización de los estudiantes de la carrera. En segunda instancia, seleccionó los programas de postgrado de la UPB más pertinentes para la profesión. A continuación, el Comité diseñó posibles rutas de formación a partir de diferentes combinaciones de estos dos componentes (campos profesionales y programas de postgrado). Por último, se establecieron criterios para escoger las rutas definitivas.

\section{Créditos del Ciclo Profesional (CP)}

Se definieron los campos en los que sería conveniente promover la especialización del estudiante de pregrado, ofreciéndole contenidos y habilidades profesionales específicos, teniendo en cuenta las siguientes fuentes: las fortalezas de la Facultad de Ingeniería Química, representadas en sus grupos de investigación: el Centro de Estudios y de Investigación en Biotecnología CIBIOT, el Grupo de Investigaciones Ambientales GIA y el grupo de investigación Pulpa y Papel; los hallazgos de las tendencias del medio (incluido el local y el regional) en los campos de desempeño profesional de los ingenieros químicos obtenidas en los estudios prospectivos realizados por la UPB (2009a); la permanente retroalimentación obtenida de egresados y/o empleadores a través de la Coordinación de prácticas de la Facultad; y el rastreo bibliográfico presentado previamente en la sección La formación en Ingeniería Química hoy. Los campos profesionales elegidos se muestran en la segunda columna de la Tabla 1. Luego se identificaron los cursos de pregrado existentes en la oferta de la Escuela de Ingenierías orientados hacia dichos campos, y en caso de que no existieran en la 
oferta, se bosquejaron los cursos precisados. Los cursos identificados conformaron lo que se ha denominado el componente del ciclo profesional.

\section{Créditos del Ciclo de Integración (Cl)}

Para conformar el segundo componente de las rutas de formación optativas, se estudiaron los diferentes postgrados, a nivel de maestría, ofrecidos por la Escuela de Ingenierías de la UPB (2015). En la Tabla 1, como ya se mencionó se presentan los campos profesionales en la segunda columna, mientras en la tercera columna se registran las maestrías seleccionadas como las más cercanas al quehacer de un ingeniero químico.

\section{Criterios para el diseño de las rutas de formación optativas}

Se analizaron las combinaciones posibles entre créditos del $\mathrm{CP}$ y créditos del $\mathrm{Cl}$ para formular rutas de formación optativas que cumplieran los siguientes criterios: Pertinencia, coherencia curricular, ajuste a las fortalezas de la Facultad Ingeniería Química y de la Escuela de Ingenierías, desarrollo de capacidades para el trabajo interdisciplinario en el estudiante, diversidad dentro del conjunto y fomento a la sostenibilidad financiera del programa de pregrado en Ingeniería Química y de la Escuela de Ingenierías.

\section{Características generales de las rutas optativas}

Para cada una de las rutas se propuso una oferta de cursos para su componente del CP tal que el estudiante tenga cierto margen de posibilidad de escoger cómo desea cubrir los 6 créditos correspondientes a este componente; es decir, la oferta completa del ciclo profesional para cada ruta excede los 6 créditos. Lo mismo se hizo para el componente del $\mathrm{Cl}$ de cada ruta, cuya oferta de cursos excede los 9 créditos, de manera que el estudiante pueda escoger cómo satisfacer este requerimiento.

Tabla 1: Campos profesionales y postgrados identificados para elaborar las rutas de formación optativas.

\begin{tabular}{|c|c|c|}
\hline & Campos profesionales & Postgrados \\
\hline $\begin{array}{c}\text { Fortalezas } \\
\text { Facultad IQ UPB }\end{array}$ & $\begin{array}{c}\text { Bioprocesos } \\
\text { Química ambiental } \\
\text { Materiales lignocelulósicos }\end{array}$ & $\begin{array}{c}\text { Maestría en Ingeniería - Área Ingeniería Química } \\
\text { Maestría en Ingeniería- Área Ambiental } \\
\text { Maestría en Diseño y Gestión de Procesos Industriales } \\
\text { Maestría en Biotecnología }\end{array}$ \\
\hline $\begin{array}{c}\text { Fortalezas } \\
\text { Escuela } \\
\text { Ingenierías UPB }\end{array}$ & $\begin{array}{c}\text { Alimentos } \\
\text { Administración y gestión } \\
\text { Calidad y producción }\end{array}$ & $\begin{array}{c}\text { Maestría en Ingeniería - Área Nuevos Materiales } \\
\text { Maestría en Ingeniería - Área Sistemas Energéticos } \\
\text { Maestría en Gestión Tecnológica }\end{array}$ \\
\hline
\end{tabular}

La selección de una ruta específica será realizada por estudiantes que hayan cursado aproximadamente el $60 \%$ del total de créditos del plan de estudios. Una vez escogida e iniciada, la ruta toma el carácter de obligatoria, y debe ser continuada y finalizada. Por último, se propone que las rutas no tengan un carácter definitivo y permanente, es decir, se actualizarán periódicamente según una caracterización de la empleabilidad del egresado de ingeniería química, salvaguardando su pertinencia.

\section{RUTAS DE FORMACIÓN OPTATIVAS DISEÑADAS}

Siguiendo el procedimiento descrito en la Metodología, se definió una oferta de nueve rutas de formación optativas para el nuevo plan de estudios de ingeniería química, cada una de las cuales tiene un componente de 6 créditos optativos del CP y un componente de 9 créditos del $\mathrm{Cl}$. La tabla 2 presenta, para cada ruta, los cursos optativos del CP y del $\mathrm{Cl}$ entre los que puede elegir un estudiante para completar los 15 créditos totales. La primera sección de la tabla muestra las cinco rutas donde los cursos del componente del CP son ofrecidos por la Facultad de Ingeniería Química (desde el pregrado en Ingeniería Química), mientras la segunda sección expone las cuatro rutas donde los cursos de pregrado del componente del CP son ofertados por otras Facultades de la Escuela de Ingenierías (desde otros pregrados en ingeniería).

Las rutas de formación optativas que se presentan entrarán en pleno funcionamiento con el nuevo plan de estudio de ingeniería química que inaugurará la UPB próximamente. Por lo pronto, la Facultad ha incursionado en ofrecer los cursos del $\mathrm{Cl}$ seleccionados para conformar las rutas a los estudiantes que cursan el plan de estudios actual (bajo la figura del $\mathrm{Cl}$ vigente al día de hoy), para detectar la acogida de la oferta entre los estudiantes, verificar los nuevos cursos delineados y perfeccionar la experiencia de contar con estudiantes de pregrado cursando asignaturas de postgrado. El resultado ha sido positivo. 
Tabla 2: Rutas de formación optativas

\begin{tabular}{|c|c|c|c|}
\hline & \multirow{3}{*}{$\begin{array}{c}\text { Rutas de Formación } \\
15 \text { créditos }\end{array}$} & $\begin{array}{c}\text { Componente Ciclo Profesional } \\
6 \text { créditos }\end{array}$ & $\begin{array}{l}\text { Componente Ciclo de Integración } \\
9 \text { créditos }\end{array}$ \\
\hline & & \multicolumn{2}{|c|}{ Opciones de cursos para completar el total de créditos de cada componente } \\
\hline & & Cursos de Pregrado & Cursos de Maestría \\
\hline \multirow{5}{*}{ 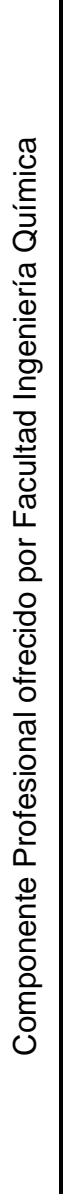 } & $\begin{array}{l}\text { Ingeniería de } \\
\text { Bioprocesos }\end{array}$ & $\begin{array}{l}\text { Bioprocesos en industria } \\
\text { alimentos } \\
\text { Bioprocesos en industria química } \\
\text { Bioprocesos en la agroindustria }\end{array}$ & $\begin{array}{l}\text { Maestría Biotecnología } \\
\text { Coloides y termodinámica de interfases } \\
\text { Biotecnología ambiental } \\
\text { Industria y procesos biotecnológicos } \\
\text { Biominería } \\
\text { Bioseparaciones }\end{array}$ \\
\hline & $\begin{array}{l}\text { Medio Ambiente y } \\
\text { Energía }\end{array}$ & $\begin{array}{l}\text { Evaluación de la calidad del agua } \\
\text { Huella ambiental } \\
\text { Calidad y contaminación de } \\
\text { suelos } \\
\text { Diagnóstico y control de } \\
\text { emisiones atmosféricas } \\
\text { Gestión de residuos } \\
\text { Valorización de lodos }\end{array}$ & $\begin{array}{l}\text { Maestría Ingeniería - Área Sistemas Energéticos } \\
\text { Gestión de la energía } \\
\text { Análisis energético sectorial } \\
\text { Perspectiva y prospectiva de los recursos } \\
\text { energéticos } \\
\text { Optimización y diseño de sistemas }\end{array}$ \\
\hline & $\begin{array}{l}\text { Aprovechamiento y } \\
\text { Valorización de } \\
\text { Residuos } \\
\text { Lignocelulósicos }\end{array}$ & $\begin{array}{l}\text { Química de materiales fibrosos } \\
\text { Conversión de biomasa vegetal } \\
\text { Biocombustibles }\end{array}$ & $\begin{array}{l}\text { Maestría Ingeniería - Área Ambiental } \\
\text { Diagnóstico y Control de la calidad del agua } \\
\text { Diagnóstico y Control de la calidad del aire } \\
\text { Diagnóstico y gestión de residuos } \\
\text { Minimización y control en la fuente } \\
\text { Gestión de residuos peligrosos } \\
\text { Química verde }\end{array}$ \\
\hline & Nuevos Materiales & $\begin{array}{l}\text { Química de materiales fibrosos } \\
\text { Conversión de biomasa vegetal } \\
\text { Biocombustibles }\end{array}$ & $\begin{array}{l}\text { Maestría Ingeniería - Área Nuevos Materiales } \\
\text { Estructura de los materiales } \\
\text { Caracterización de los materiales } \\
\text { Propiedades de materiales } \\
\text { Selección de materiales } \\
\text { Plásticos o transformación de fase }\end{array}$ \\
\hline & $\begin{array}{l}\text { Modelamiento y } \\
\text { Simulación de } \\
\quad \text { Procesos }\end{array}$ & $\begin{array}{l}\text { Volúmenes finitos } \\
\text { Termofluidos computacionales }\end{array}$ & $\begin{array}{l}\text { Maestría Ingeniería - Área Ingeniería Química } \\
\text { Termodinámica avanzada } \\
\text { Fenómenos de transporte avanzados } \\
\text { Ingeniería de las reacciones químicas }\end{array}$ \\
\hline \multirow{4}{*}{ 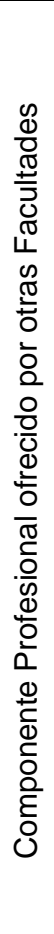 } & $\begin{array}{l}\text { Ingeniería y Gestión } \\
\text { de Procesos }\end{array}$ & $\begin{array}{l}\text { Pregrado Ingeniería Mecánica } \\
\text { Planeación y control de la } \\
\text { producción } \\
\text { Pregrado Ingeniería Industrial } \\
\text { Logística industrial } \\
\text { Pregrado Ingeniería Agroindustrial } \\
\text { Aseguramiento de la calidad } \\
\end{array}$ & $\begin{array}{l}\text { Maestría Diseño y Gestión de Procesos Industriales } \\
\text { Optimización de procesos } \\
\text { Control de procesos } \\
\text { Seguridad y Prevención de pérdidas } \\
\text { Gestión Ambiental y Energética }\end{array}$ \\
\hline & $\begin{array}{l}\text { Industria de } \\
\text { Alimentos y Medio } \\
\text { Ambiente }\end{array}$ & $\begin{array}{l}\text { Pregrado Ingeniería Agroindustrial } \\
\text { Tecnología Alimentaria I: Lácteos } \\
\text { Tecnología Alimentaria II: } \\
\text { Cárnicos } \\
\text { Tecnología Alimentaria III: } \\
\text { Vegetales }\end{array}$ & $\begin{array}{l}\text { Maestría Ingeniería- Área Ambiental } \\
\text { Diagnóstico y Control de la calidad del agua } \\
\text { Diagnóstico y Control de la calidad del aire } \\
\text { Diagnóstico y gestión de residuos } \\
\text { Minimización y control en la fuente } \\
\text { Gestión de residuos peligrosos } \\
\text { Química verde }\end{array}$ \\
\hline & $\begin{array}{c}\text { Economía y Gestión } \\
\text { Tecnológica }\end{array}$ & $\begin{array}{l}\text { Pregrado Ingeniería Agroindustrial } \\
\text { Costos y presupuestos } \\
\text { Economía } \\
\text { Mercados }\end{array}$ & $\begin{array}{l}\text { Maestría Gestión Tecnológica } \\
\text { Gestión del conocimiento } \\
\text { Prospectiva tecnológica } \\
\text { Transferencia de tecnología } \\
\text { Modelos cuantitativos de decisión }\end{array}$ \\
\hline & $\begin{array}{l}\text { Nuevos Materiales } \\
\text { y Nanotecnología }\end{array}$ & $\begin{array}{l}\text { Pregrado Ingeniería en } \\
\text { Nanotecnología } \\
\text { Nanotecnología I } \\
\text { Nanotecnología II }\end{array}$ & $\begin{array}{l}\text { Maestría Ingeniería - Área Nuevos Materiales } \\
\text { Caracterización de materiales } \\
\text { Propiedades de los materiales } \\
\text { Estructura de los materiales } \\
\text { Procesado de materiales compuestos }\end{array}$ \\
\hline
\end{tabular}


Para finalizar, es pertinente señalar que la propuesta de la UPB para las diversas especializaciones en la carrera de ingeniería química contiene los temas más frecuentes en las ofertas del mismo tipo de diferentes universidades, tal como se reseñó en este artículo: medio ambiente y sostenibilidad (incluyendo el tema de la energía), materiales y biotecnología. Pero además presenta para los estudiantes del pregrado, como elemento diferenciador, la posibilidad de especializarse en cuestiones como modelamiento y simulación de procesos, ingeniería y gestión de procesos, economía y gestión tecnológica, y nanotecnología, en el marco del estudio de los nuevos materiales.

\section{CONCLUSIONES}

Las rutas de formación optativas diseñadas para la carrera en Ingeniería Química de la UPB cumplen variados propósitos: habilitan al estudiante para desempeñarse con éxito en campos profesionales y ocupacionales específicos; facilitan la integración del futuro profesional en ingeniería química a un estudio de postgrado; contribuyen a la flexibilidad del currículo, abriendo espacios para que los estudiantes decidan sobre la orientación de su educación en razón de sus capacidades y sus intereses; y potencian la capacidad de trabajo interdisciplinar de la comunidad académica.

Las rutas de formación optativas expuestas contienen temas que coinciden con los incluidos en la oferta de especialización de la carrera de ingeniería química de otras universidades de la región y del mundo (medio ambiente, materiales, energía, biotecnología); pero también despliegan temáticas diferentes y pertinentes para los futuros ingenieros químicos: modelamiento y simulación de procesos, ingeniería y gestión de procesos, economía y gestión tecnológica, y nanotecnología.

La oferta de las rutas de formación optativas presentada será dinámica, se revisará periódicamente para garantizar que permanezca acorde al entorno profesional -nacional y mundial- en el que se desempeñan los ingenieros químicos.

\section{REFERENCIAS}

Allen, D.T. y Shonnard, D.R., Sustainability in chemical engineering education: identifying a core body of knowledge, AIChE Journal: 58(8), 2296-2302 (2012)

Armstrong, R.C., A vision of the chemical engineering curriculum of the future, Chemical engineering education: 40(2), 104-110 (2006)

Assaf-Anid, N.M. y Hollein, H.C., Incorporating biotechnology in chemical engineering curriculum, Proceedings of the 2002 American Society for Engineering Education Annual conference \& Exposition, Montreal, Canada, 16-19 de junio (2002)

Byrne, E.P., The role of specialization in the chemical engineering curriculum, Education for Chemical Engineers - Official Journal of the European Federation of Chemical Engineering: Part D, 1(1), 3-15 (2006)

Carmona, L.F., Benchmarking comparativo de los Programas de Pregrado en Ingeniería Química en países miembros de la OEA, Tesis de Maestría en Gestión Tecnológica, Sistema de Formación Avanzada, Universidad Pontificia Bolivariana, Medellín, Colombia (2014)

Castrillón, F. y Garcés, B., Camino y logros de una transformación curricular en Ingeniería Química, World Engineering Education Forum - WEEF 2012, Buenos Aires, Argentina, 15-18 octubre (2012), http://www.weef2012.edu.ar/papersFinal/information.php?doc=439. Acceso: 1 de febrero (2015)

Charpentier, J.-C., Four main objectives for the future of chemical and process engineering mainly concerned by the science and technologies of new materials production, Chemical engineering journal: 107(1-3), 3-17 (2005)

Cortese, A. D., The critical role of higher education in creating a sustainable future, Planning for higher education: $31(3), 15-22(2003)$

Crawley, E., Malmqvist, J., Ostlund, S. y Brodeur, D., Rethinking engineering education: The CDIO approach, 234-235, Springer, Nueva York, Estados Unidos de América (2007)

Gillett, J.E., Chemical Engineering Education in the Next Century, Chemical Engineering Technology: 24(6), 561-570 (2001) 
IChemE - Institution of Chemical Engineers, A Roadmap for 21st Century chemical engineering, 1-49, IChemE, Londres, Reino Unido (2007)

León, V.A. y Lugo, E., Curricular innovation and emerging knowledge in chemical engineering in Mexico. Study comparative, doi: 10.1016/j.sbspro.2015.01.1006, Procedia - Social and Behavioral Sciences (en línea), 174, 3374-3377 (2015)

Molzahn, M., Chemical engineering education in Europe: Trends and challenges, Chemical Engineering Research and Design: 82(12), 1525-1532 (2004)

República de Colombia - Ministerio de Educación, Decreto 1295 de abril 20 de 2010 por el cual se reglamenta el registro calificado de que trata la Ley 1188 de 2008 y la oferta y desarrollo de programas académicos de educación superior, Ministerio de Educación, Bogotá, Colombia (2010)

UPB - Universidad Pontificia Bolivariana, Estudio Prospectiva para la Ingeniería Química 2019, UPB, Medellín, Colombia (2009a)

UPB - Universidad Pontificia Bolivariana, Modelo pedagógico integrado, UPB, Medellín, Colombia (2009b), http://www.upb.edu.co/pls/portal/docs/PAGE/GP_REPOSITORIO_IMAGENES/PG_RIMG_MEDELLIN/MOD ELO\%20PEDAGOGICO.PDF. Acceso: 11 de noviembre (2014)

UPB - Universidad Pontificia Bolivariana, Proceso de transformación curricular. Orientaciones Metodológicas. La distribución de los contenidos curriculares. Tercera Parte, UPB, Medellín, Colombia (2011a)

UPB - Universidad Pontificia Bolivariana, Proceso de transformación curricular. Orientaciones Metodológicas. La organización. Segunda Parte, UPB, Medellín, Colombia (2011b)

UPB - Universidad Pontificia Bolivariana, Proceso transformación curricular. Orientaciones Metodológicas. Primera Parte, UPB, Medellín, Colombia (2011c)

UPB - Universidad Pontificia Bolivariana, Programas Escuela de Ingenierías (ver Maestrías), en línea, Medellín,

UPB, http://www.upb.edu.co/portal/page?_pageid=1054,32103302\&_dad=portal\&_schema=PORTAL. Acceso: 1 de febrero (2015) 
